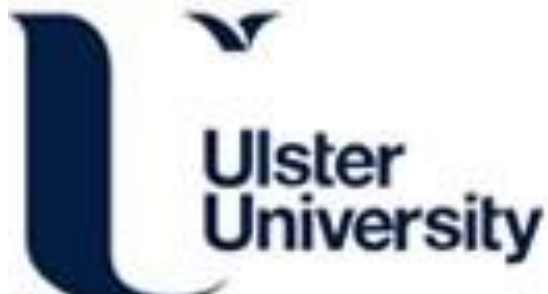

\section{Improving the Signal-To-Noise Ratio When Monitoring Countermovement Jump Performance}

Kennedy, R., \& Drake, D. (2018). Improving the Signal-To-Noise Ratio When Monitoring Countermovement Jump Performance: Signal-to-noise ratio of CMJ performance. Journal of Strength and Conditioning Research. https://doi.org/10.1519/JSC.0000000000002615

Link to publication record in Ulster University Research Portal

Published in:

Journal of Strength and Conditioning Research

Publication Status:

Published online: 08/05/2018

DOI:

10.1519/JSC.0000000000002615

Document Version

Author Accepted version

\section{General rights}

Copyright for the publications made accessible via Ulster University's Research Portal is retained by the author(s) and / or other copyright owners and it is a condition of accessing these publications that users recognise and abide by the legal requirements associated with these rights.

\section{Take down policy}

The Research Portal is Ulster University's institutional repository that provides access to Ulster's research outputs. Every effort has been made to ensure that content in the Research Portal does not infringe any person's rights, or applicable UK laws. If you discover content in the Research Portal that you believe breaches copyright or violates any law, please contact pure-support@ulster.ac.uk. 
Manuscript Title: Improving the signal-to-noise ratio when monitoring countermovement jump performance

Running Head: Signal-to-noise ratio of CMJ performance

Laboratory: Ulster Rugby, Kingspan Stadium, Belfast, N. Ireland

Authors: Rodney A. Kennedy ${ }^{1 *}$ and David Drake ${ }^{2}$

Department/Institution: ${ }^{1}$ School of Sport, Ulster University, Jordanstown, N. Ireland; ${ }^{2}$ Ulster Rugby, Kingspan Stadium, Belfast, N. Ireland

Corresponding Author*: School of Sport, Ulster University, Jordanstown, Shore Road, Newtownabbey, Co. Antrim, BT37 0QB, N. Ireland.

Phone: +442890366242

Email: r.kennedy@ulster.ac.uk

Funding: No external financial support was received for this study 


\begin{abstract}
Countermovement jump (CMJ) performance has been routinely used to monitor neuromuscular status. However, the protocol used to establish the criterion score is not well documented. The purpose of this study to examine how the protocol used would influence of the sensitivity of CMJ variables in rugby union players. Fifteen male (age: $19.7 \pm 0.5$ years) rugby union players performed $8 \mathrm{CMJs}$ on two occasions, separated by 7-days. The between session coefficient of variation $(\mathrm{CV})$ was calculated using two techniques for treating multiple trials, the average and the trial with the best jump height $(\mathrm{JH})$, and then compared to the smallest worthwhile change (SWC). The signal-to-noise ratio was measured as the group mean change in a variable divided by the CV. Using the average value across multiple trials is superior to the best trial method, based on lower CVs for all variables. Only the average performance across 6 or more trials was classified as ideal $(\mathrm{CV}<0.5 \mathrm{x}$ SWC) for peak velocity (PV). In addition, the signal-to-noise ratio for peak concentric power (PCP), PV and $\mathrm{JH}$ were classified as good, irrespective of the treatment method. Although increasing the number of trials can reduce the random error, it may be pragmatic to simply take the average from 2-3 trials, facilitating a CV $<$ SWC for PV, PCP and JH. Due to its simplicity, JH may be considered the principal variable to monitor neuromuscular fatigue.
\end{abstract}

KEYWORDS: athletic performance, movement, sensitivity, probability 


\section{INTRODUCTION}

Countermovement jump (CMJ) performance has been used for many decades to examine the effects of a wide variety of training modalities. In recent years, these protocols have also been used to provide a functional measure of neuromuscular fatigue after both training and competition. Collectively, the kinetic and kinematic variables used have provided sensitive markers in many, but not all investigations $(4,29)$. The disparity in the findings reported have been ascribed to several factors, with the protocol used to establish the criterion score being a confounding factor that has not been well documented. While several authors have used the results from a single trial $(6,19)$, the variability that is intrinsic to all human movement suggests that such an approach may not adequately represent an athletes' performance. Increasing the number of trials may potentially overcome this concern; however, it needs to be recognised that it may not be practical or physically possible to collect multiple trials in certain situations. On most occasions, athletes are required to perform 2-3 trials, with as many as 8 trials sometimes being advocated (5). It is notable that previous studies have not provided a clear rationale for the number of trials used and the decisions seem somewhat arbitrary in nature.

Two techniques for treating multiple trials have been identified: best or taking the average. The single best trial is most commonly retained for analysis (4) and of great interest to many coaches. When both techniques were used in the same training study, the ability to monitor changes in CMJ performance over time was viewed as comparable and likely to produce similar outcomes, at both the group and individual level (11). However, a recent metaanalysis found average, rather than best CMJ performance, to be more sensitive when monitoring changes in neuromuscular status (4). Furthermore, despite the popularity of using 
CMJ performance as an objective marker of neuromuscular performance, there is a lack of consensus regarding the most sensitive variable. The height jumped, is the most commonly used variable, which seems well founded based on the outcomes of a meta-analytic approach $(4,29)$. However, several studies have reported $(6,10,27,33)$, that in certain situations, jump height $(\mathrm{JH})$ was not a sensitive measure of fatigue and as such, advocated a focus on the movement strategy used to achieve the output.

A potential solution to address these aforementioned issues, which is often overlooked within strength and conditioning (18), is to consider the signal-to-noise ratio of the derived CMJ variables. When the noise is expressed as a coefficient of variation $(\mathrm{CV})$, it has often been used in isolation with arbitrary thresholds, such as $5 \%$ and $10 \%$, to define an acceptable margin of error. Such an approach allows the coaches to assess changes relative to the expected error values but does not provide any information regarding the meaningfulness of the change. Coaches initially want to ascertain the likelihood that the changes observed are greater than the smallest signal for their specific population, referred to as the smallest worthwhile change (SWC) (17). To facilitate this process, it requires a test or variable with a $\mathrm{CV}<\mathrm{SWC}$, with the ideal contrast being when the CV $<0.5 \mathrm{x}$ SWC, thus enabling any change that is greater than the SWC being classified as substantial $(3,15)$. It is well established that increasing the number of trials will theoretically reduce the CV by a factor of $1 / \sqrt{ }$ n (i.e., using 4 trials reduces the noise by $50 \%$ ) (15). Nonetheless, a variable with a relatively low level of noise doesn't always constitute an effective tool to monitor fatigue and supercompensation $(3,30)$. The noise also needs to be compared to the observed signal, to provide another important index of sensitivity (18). Therefore, the aim of this study was to consider how the protocol used to establish the criterion score would influence the sensitivity of countermovement jump performance in rugby union players. As a working hypothesis, we 
assumed that the average of multiple trials would decrease the noise associated with a CMJ variable and as a result, improve the signal-to-noise ratio for the main outcome variable, jump height.

\section{METHODS}

\section{Subjects}

Fifteen male academy players from a professional rugby union club in the United Kingdom volunteered to take part in the study (age $19.7 \pm 0.5$ years, height $185.2 \pm 7.5 \mathrm{~cm}$, and mass $97.0 \pm 10.6 \mathrm{~kg})$. Although this sample size is congruent with similar studies $(26,30)$, more subjects would be required to attain optimal precision for estimates of reliability and as such, this study can be viewed as a preliminary investigation (14). To be eligible for inclusion in the study, players must have been part of academy squad at the start of the playing season and therefore familiar with the CMJ testing that is routinely conducted. Exclusionary criteria included players with known musculoskeletal injury or pain during the time of testing. The weekly training volume of the players was 8-12 hours (5-7 hours of rugby training, 1-2 hours of speed and agility training, plus 2-3 hours of gym based preparation), and one competitive game. Prior to the study commencing, the players attended a presentation to outline the purpose, benefits, risks and procedures involved in the study. Players provided written informed consent and were free to withdraw from the study at any stage without penalty. The study was approved by the Ulster University Human Research Ethics Committee.

\section{Design}

A within-subject test-retest design was used, with the testing protocol completed on two occasions, separated by 7 days. To control for the possible influence of circadian rhythms 
and the phase of training, all tests were performed during the morning period of the competitive season. Subjects were asked to maintain their normal diet throughout and to refrain from strenuous exercise 48 hours before each session. Prior to the tests, subjects completed a 10-minute standardised warm-up consisting of jogging, dynamic stretching, and several practice jumps of progressively increasing intensity until they felt capable of producing a maximal effort.

\section{Methodology}

CMJ trials were performed on a force plate (Type 9286BA, Kistler AG, Winterthur, Switzerland) that was connected to an A/D convertor (Type 5691A1, Kistler AG, Winterthur, Switzerland). Temporal and vertical ground reaction force $\left(F_{\mathrm{z}}\right)$ data were collected at a sampling frequency of $1000 \mathrm{~Hz}$ for 5 seconds using Bioware ${ }^{\circledR}$ software (Version 5.1, Type 2812A). The force plate was zeroed immediately before each trial and sampling began when the subject was standing still. After approximately 2 seconds, subjects were instructed to jump as high as possible using a self-determined countermovement depth. Each subject completed 8 trials with 1 minute of rest between. The raw $F_{\mathrm{z}}$ data for each jump were exported as text files and analysed using a customized Microsoft Excel ${ }^{\circledR}$ spreadsheet. The subjects' body weight was calculated as the average $F_{\mathrm{z}}$ during the first second of the sampling period. The start of the countermovement was determined as $5 \%$ reduction in $F_{\text {z. }}(7)$ The take-off and landing time points were determined by finding the 0.4 second moving average with the smallest standard deviation $F_{\mathrm{z}}$ and then taking the peak residual force during this phase as the threshold. The vertical velocity of the centre of mass (COM) was determined using the impulse method. Net impulse was obtained by integrating net $F_{\mathrm{z}}$ using the trapezoid method from the start of the countermovement and then dividing it by body mass to obtain vertical velocity. Although not always advocated (34), power was calculated as the product of $F_{\mathrm{z}}$ and velocity, to allow comparison with other commonly used variables. The vertical 
velocity of the COM at take-off was used to calculate JH. The eccentric and concentric phases were defined as: eccentric phase, the start of the countermovement to the transition from negative to positive velocity; concentric phase, the end of the eccentric phase to the point of take-off. The reactive strength index-modified (RSImod) was calculated as the JH divided by the contraction time (9). The flight time:contraction time ratio (FT:CT) was calculated as previously described (7).

\section{Statistical Analysis}

A customized Microsoft Excel ${ }^{\circledR}$ spreadsheet (16) was used to determine the typical error (TE), calculated as the standard deviation of the difference score divided by $\sqrt{ } 2$ and expressed as a coefficient of variation (CV) with $\pm 90 \%$ confidence intervals (CI). The SWC was calculated as 0.2 times the between subject SD, which corresponds to a small effect, and expressed as a percentage of the mean to compare with the CV (12). The usefulness of a variable was rated as marginal when the $\mathrm{CV}>\mathrm{SWC}$, okay when the $\mathrm{CV}=\mathrm{SWC}$, good when the $\mathrm{CV}<\mathrm{SWC}(15)$, and ideal when the $\mathrm{CV}<0.5 \mathrm{x}$ SWC $(3,15)$. To examine the influence of the number of trials, the between session $\mathrm{CV}$ was calculated using the two techniques for treating multiple trials, the average and the trial with the best $\mathrm{JH}$. The sensitivity to change $\pm 90 \% \mathrm{CI}$, sometimes referred to as internal responsiveness (18), was calculated by dividing the signal by the noise. The percentage change in the group mean between baseline and 48 hours post-exercise was considered as the signal, provided by a previous similar study (20), and the $\mathrm{CV}$ from 3 trials as the noise, provided from the current study. The utility of a signalto-noise ratio was classified as good when $>1$, okay if $\approx 1$, and poor if $<1$ (8). 


\section{RESULTS}

Figure 1 illustrates the estimates of within-subject variation for the two treatment techniques of multiple trials, the average and the trial with the best $\mathrm{JH}$, relative to the SWC and $0.5 \mathrm{x}$ SWC. Without exception, the average of multiple trials resulted in lower CVs when compared to the best. PV, PCP and JH were classified as good (CV < SWC) when the average of 2 or more trials were performed. Using the best trial method, PV and JH were classified as good when 3 or more trials were performed. Only the average performance across 6 or more trials was classified as ideal (CV $<0.5 \mathrm{x}$ SWC) for PV. All other variables were classified as marginal $(\mathrm{CV}>\mathrm{SWC})$.

***FIGURE 1 NEAR HERE AS A 2 X 3 MATRIX***

Figure 2 illustrates that the signal-to-noise ratios for PV, PCP and JH were classified as good, irrespective of the treatment method but were consistently higher for the average method. All other variables were classified as poor. PV, using the average method, had the highest (2.9 $\pm 1.2)$ signal-to-noise ratio, with PCP $(2.5 \pm 1.3)$ and JH $(2.4 \pm 1.1)$ having comparable values.

\section{***FIGURE 2 NEAR HERE***}

\section{DISCUSSION}

CMJ performance is routinely used to monitor fatigue and supercompensation in athletic populations. Considering that it is viewed essential to identify the true value when 
determining a real and meaningful change, we investigated the sensitivity of CMJ variables using two techniques for treating multiple trials: best or taking the average. The main findings of the present study indicated that average performance across multiple trials is superior to the best trial method, as determined by lower CVs (Figure 1). These results are in accordance rudimentary reliability theory, in which multiple trials will reduce the noise associated with a variable and thus produce a better estimate of performance. When trying to identify a variable that could measure a change that is greater than the SWC, only the average PV across 6 trials was classified as ideal $(\mathrm{CV}<0.5 \mathrm{x}$ SWC). In addition, the signal-to-noise ratio was classified as good for $\mathrm{PV}, \mathrm{PCP}$ and $\mathrm{JH}$, irrespective of the treatment method but were consistently higher for the average method (Figure 2).

When multiple trials of a test are collected, then the decision regarding which trial or trials to use as the criterion score represents a long forgotten question, as evidenced by the fact that the vast majority of published studies having used the best CMJ (4). It has been recommended that the selection should be based on whether the criterion score is supposed to reflect the typical or maximum performance (13). Therefore, it appears evident that the average method should be used to determine the criterion score if an estimate of typical CMJ performance is required. In a sport like track and field athletics, the best result is understandably used in multiple trial events such as the long jump or shot put, because maximum performance is the measure of success. However, by definition the best performance is not typical and may demonstrate greater variability, as presented in Figure 1, and this choice makes as much sense as selecting the lowest score when trying to monitor typical performance (21). It would seem that almost all researchers and coaches are looking for an estimate of typical CMJ performance and are therefore ill-advised to disregard large amounts of data by selecting the best trial. 
Increasing the number of trials will in most cases reduce the typical error associated with a test variable. However, the number of trials required to achieve a desirable level $(\mathrm{CV}<\mathrm{SWC})$ has not been well documented (30). Taking the average across 8 trials reduces the CV by approximately $1-3 \%$, depending on the variable. Although it may be possible to reduce the random error, the additional time required needs to be carefully considered by the coach. Unless a scenario exists that requires detection of the SWC, then it may be advisable to simply take the average from 2-3 trials, to facilitate a $\mathrm{CV}<\mathrm{SWC}$ for $\mathrm{PV}, \mathrm{PCP}$ and $\mathrm{JH}$. The output of a CMJ can be considered very reliable, but there is a tremendous amount of redundancy in the neuromuscular system that permits a multitude of ways in which a JH can be achieved, even in a non-fatigued state. It is therefore virtually impossible to repeat a CMJ in the exact same manner and for that reason, a major source of error in composite variables, such as FT:CT and RSImod, can be attributed to the biovariance inherent in all human movement (25).

Despite the popularity of using CMJ performance as an objective marker of fatigue or supercompensation, there is no consensus on which variable is the most sensitive. Intuitively, coaches are always looking for variables that exhibit low levels of noise relative to the smallest signal $(\mathrm{CV}<\mathrm{SWC})$. Nonetheless, it needs to be appreciated that even when this condition is met, it does not automatically constitute a variable that can effectively monitor fatigue and supercompensation $(3,30)$. The signal-to-noise ratio of a test is viewed as an essential, but largely overlooked property within strength and conditioning (18). A variable, such as peak concentric force (PCF), that exhibits a relatively low CV but is also unresponsive to fatigue inducing exercise $(19,32)$, doesn't represent a useful variable to 
monitor (Figures $1 \& 2$ ). The magnitude of the signal-to-noise ratio can therefore be considered of paramount importance to every coach (3). JH is the most commonly used outcome variable $(4,29)$, which seems well founded, based on the signal-to-noise ratio (Figure 2). In contrast, several studies have reported that impairments in neuromuscular function can manifest themselves as an altered movement strategy in an attempt to achieve a constant task outcome, such as $\mathrm{JH}(6,10,27,33)$. The disparity in the findings can be attributed to not using fatigue-free baseline values (6), combining force plate and linear position transducer data $(10,24)$, and the threshold adopted to denote a substantial change $(27,33)$. Future research may want to consider the effect of countermovement depth at the fatigue-free baseline time-point on the response to fatigue. It may be well be that certain individuals that adopt a relatively small amplitude CMJ are more likely to demonstrate an altered movement strategy in response to fatigue, based on the relationship between countermovement depth and $\mathrm{JH}(22)$.

Although this study presents some novel findings, there are a few limitations that the practitioner should keep in mind when interpreting the findings. Firstly, increasing the sample size would help attain optimal precision for the estimates of reliability and therefore this study can be viewed as a preliminary investigation into the area (14). Another limitation to consider when using the between subject SD to provide an indirect estimate of the SWC, is that the value calculated will vary depending on the sample used, a homogeneous group of players will display a correspondingly low value for the SWC (28). Finally, the signal-tonoise ratios reported are specific to the sample population $(1,26)$ and also to the nature of the fatigue inducing exercise utilised (23). 


\section{PRACTICAL APPLICATIONS}

Increasing the number of trials in a $\mathrm{CMJ}$ testing protocol will reduce the noise associated with the performance variables chosen. However, the additional time required needs to be carefully considered and it may be preferential to only take 2-3 trials. Coaches should also appreciate that when multiple trials are taken, they are ill-advised to disregard large amounts of data by selecting the best trial and should take the average. Variables should be selected from the force-time curve that have been demonstrated as sensitive, rather than obscure variables, that are unsubstantiated and do not advance jumping related research and practice. Although PV demonstrated a slightly higher signal-to-noise ratio, JH may be considered the principal variable to monitor fatigue, as it can also be estimated using a contact mat or a similar flight time based system (2,31). 


\section{REFERENCES}

1. Ahtianen, JP and Hakkinen, K. Strength athletes are capable to produce greater muscle activiation and neural fatigue during high-intensity resistance training exercise than nonathletes. J Strength Cond Res 23: 1129-1134, 2009.

2. Brownstein, CG, Dent, JP, Parker, P, Hicks, KM, Howatson, G, Goodall, S, et al. Etiology and recovery of neuromuscular fatigue after simulated soccer match play. Front Physiol 8: 1-14, 2017.

3. Buchheit, M. Monitoring training status with HR measures: Do all roads lead to Rome? Front Physiol 5: 1-19, 2014.

4. Claudino, JG, Cronin, J, Mezêncio, B, McMaster, DT, McGuigan, M, Tricoli, V, et al. The countermovement jump to monitor neuromuscular status: A meta-analysis. J Sci Med Sport 20: 397-402, 2017.

5. Claudino, JG, Mezncio, B, Soncin, R, Ferreira, JC, Couto, BP, and Szmuchrowski, LA. Pre vertical jump performance to regulate the training volume. Int J Sports Med 33: 101-107, 2012.

6. Cormack, SJ. Neuromuscular fatigue and endocrine responses in elite Australian Rules match. Int J Sports Physiol Perform 3: 359-374, 2008.

7. Cormack, SJ, Newton, RU, McGulgan, MR, and Doyle, TLA. Reliability of measures obtained during single and repeated countermovement jumps. Int J Sports Physiol Perform 3: 131-144, 2008.

8. Crowcroft, S, McCleave, E, Slattery, K, and Coutts, AJ. Assessing the measurement sensitivity and diagnostic characteristics of athlete monitoring tools in national 
swimmers. Int J Sports Physiol Perform 12: 95-100, 2017.

9. Ebben, WP and Petushek, EJ. Using the reactive strength index modified to evaluate plyometric performance. J Strength Cond Res 24: 1983-1987, 2010.

10. Gathercole, R, Sporer, B, Stellingwerff, T, and Sleivert, G. Alternative countermovement-jump analysis to quantify acute neuromuscular fatigue. Int J Sports Physiol Perform 10: 84-92, 2015.

11. Al Haddad, H, Simpson, BM, and Buchheit, M. Monitoring changes in jump and sprint performance: best or average values? Int J Sports Physiol Perform 10: 931-934, 2015.

12. Haugen, T and Buchheit, M. Sprint running performance monitoring: methodological and practical considerations. Sport Med 46: 641-656, 2016.

13. Hetherington, R. Within-subject variation, measurement error, and selection of a criterion score. Res $Q$ 44: 113-117, 1973.

14. Hopkins, WG. Measures of reliability in sports medicine and science. Sport Med 30: $1-15,2000$.

15. Hopkins, WG. How to interpret changes in an athletic performance test. Sportscience 8: 1-7, 2004.

16. Hopkins, WG. Analysis of reliability with a spreadsheet. Available at: sportsci.org/resource/stats/xrely.xls. http://sportsci.org/resource/stats/xrely.xls., 2007.

17. Hopkins, WG, Marshall, SW, Batterham, AM, and Hanin, J. Progressive statistics for studies in sports medicine and exercise science. Med Sci Sports Exerc 41: 3-12, 2009.

18. Impellizzeri, FM and Marcora, SM. Test validation in sport physiology: lessons learned from clinimetrics. Int J Sports Physiol Perform 4: 269-277, 2009. 
19. Johnston, RD, Gibson, N V., Twist, C, Gabbett, TJ, MacNay, SA, and MacFarlane, NG. Physiological responses to an intensified period of rugby league competition. $J$ Strength Cond Res 27: 643-654, 2012.

20. Kennedy, RA and Drake, D. Dissociated time course of recovery between strength and power after isoinertial resistance loading in rugby union players. J Strength Cond Res (in press), 2017.

21. Kroll, W. Reliability theory and research decision in selection of a criterion score. Res Q 38: 412-419, 1967.

22. Mandic, R, Jakovljevic, S, and Jaric, S. Effects of countermovement depth on kinematic and kinetic patterns of maximum vertical jumps. J Electromyogr Kinesiol 25: $265-272,2015$.

23. McCaulley, GO, McBride, JM, Cormie, P, Hudson, MB, Nuzzo, JL, Quindry, JC, et al. Acute hormonal and neuromuscular responses to hypertrophy, strength and power type resistane exercise. Eur J Appl Physiol 105: 695-704, 2009.

24. Mundy, PD, Lake, JP, Carden, PJC, Smith, NA, and Lauder, MA. Agreement between the force platform method and the combined method measurements of power output during the loaded countermovement jump. Sport Biomech 15: 23-35, 2016.

25. Rodano, R and Squadrone, R. Stability of selected lower limb joint kinetic parameters during vertical jump. J Appl Biomech 18: 83-89, 2002.

26. Roe, G, Darrall-Jones, J, Till, K, Phibbs, P, Read, D, Weakly, J, et al. Between-day reliability and sensitivity of common fatigue measure in rugby players. Int J Sport Physiol Perform 581-586, 2016. 
27. Rowell, AE, Aughey, RJ, Hopkins, WG, Stewart, AM, and Cormack, SJ. Identification of sensitive measures of recovery following external load from football match play. Int J Sports Physiol Perform 1-44, 2017.

28. Saunders, PU, Pyne, DB, Telford, RD, and Hawley, JA. Reliability and variability of running economy in elite distance runners. Med Sci Sports Exerc 36: 1972-1976, 2004.

29. Silva, JR, Rumpf, MC, Hertzog, M, Castagna, C, Farooq, A, Girard, O, et al. Acute and residual soccer match-related fatigue: a systematic review and meta-analysis. Sport Med 1-45, 2017.

30. Taylor, KL, Cronin, J, Gill, ND, Chapman, DW, and Sheppard, J. Sources of variability in isoinertial jump assessments. Int J Sports Physiol Perform 5: 546-558, 2010.

31. Thomas, K, Dent, J, Howatson, G, and Goodall, S. Etiology and recovery of neuromuscular fatigue after simulated soccer match play. Med Sci Sports Exerc 49: 955-964, 2017.

32. Thorlund, JB, Michalsik, LB, Madsen, K, and Aagaard, P. Acute fatigue-induced changes in muscle mechanical properties and neuromuscular activity in elite handball players following a handball match. Scand J Med Sci Sport 18: 462-472, 2008.

33. Weakley, JJS, Till, K, Read, DB, Roe, GAB, Darrall-Jones, J, Phibbs, PJ, et al. The effects of traditional, superset, and tri-set resistance training structures on perceived intensity and physiological responses. Eur J Appl Physiol 117: 1877-1889, 2017.

34. Winter, EM, Abt, G, Brookes, FBC, Challis, JH, Fowler, NE, Knudson, D V, et al. Misuse of "power" and other mechanical terms in sport and exercise science research. 
J Strength Cond Res 30: 292-300, 2016. 


\section{Figure 1.}

Coefficient of variation $\pm 90 \%$ confidence interval, relative to the SWC and $0.5 \times \mathrm{SWC}$, for peak velocity (PV), peak concentric force (PCF), peak concentric power (PCP), jump height $(\mathrm{JH})$, reactive strength index-modified (RSImod) and flight time:contraction time ratio (FT:CT) based on the number of trials and the two treatment techniques of multiple trials, the average and the best.

\section{Figure 2.}

Signal-to-noise ratio $\pm 90 \%$ confidence interval for peak velocity (PV), peak concentric force (PCF), peak concentric power (PCP), jump height $(\mathrm{JH})$, reactive strength index-modified (RSImod) and flight time:contraction time ratio (FT:CT) based on the two treatment techniques of multiple trials, the average and the best. Note that the signal-to-noise ratio was calculated as the percentage change in the group mean, provided by a previous similar study (20), and then divided by the CV from the current study using the results from trial number 3 . These results are specific to the population and the nature of the fatigue inducing exercise. 


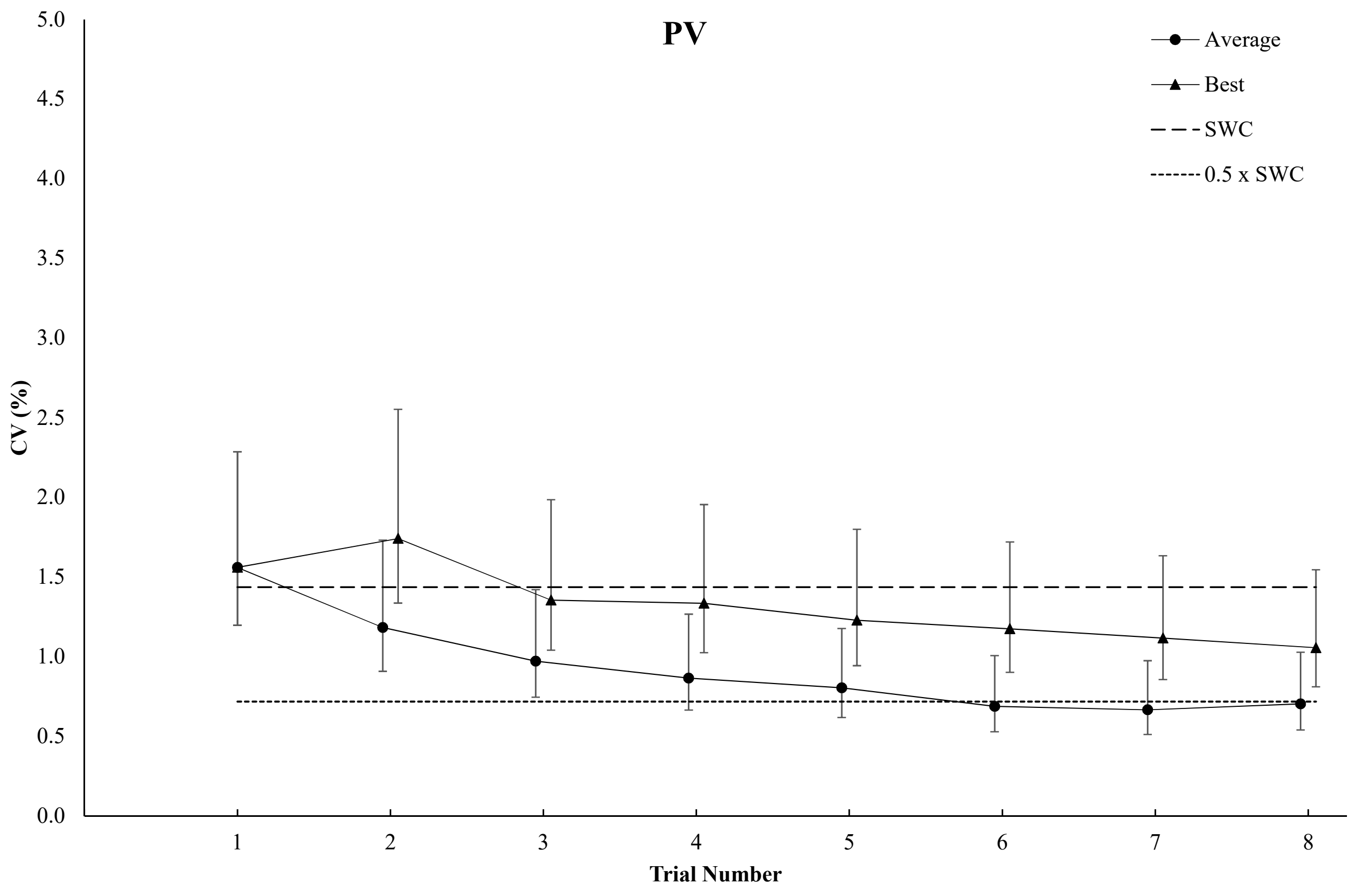




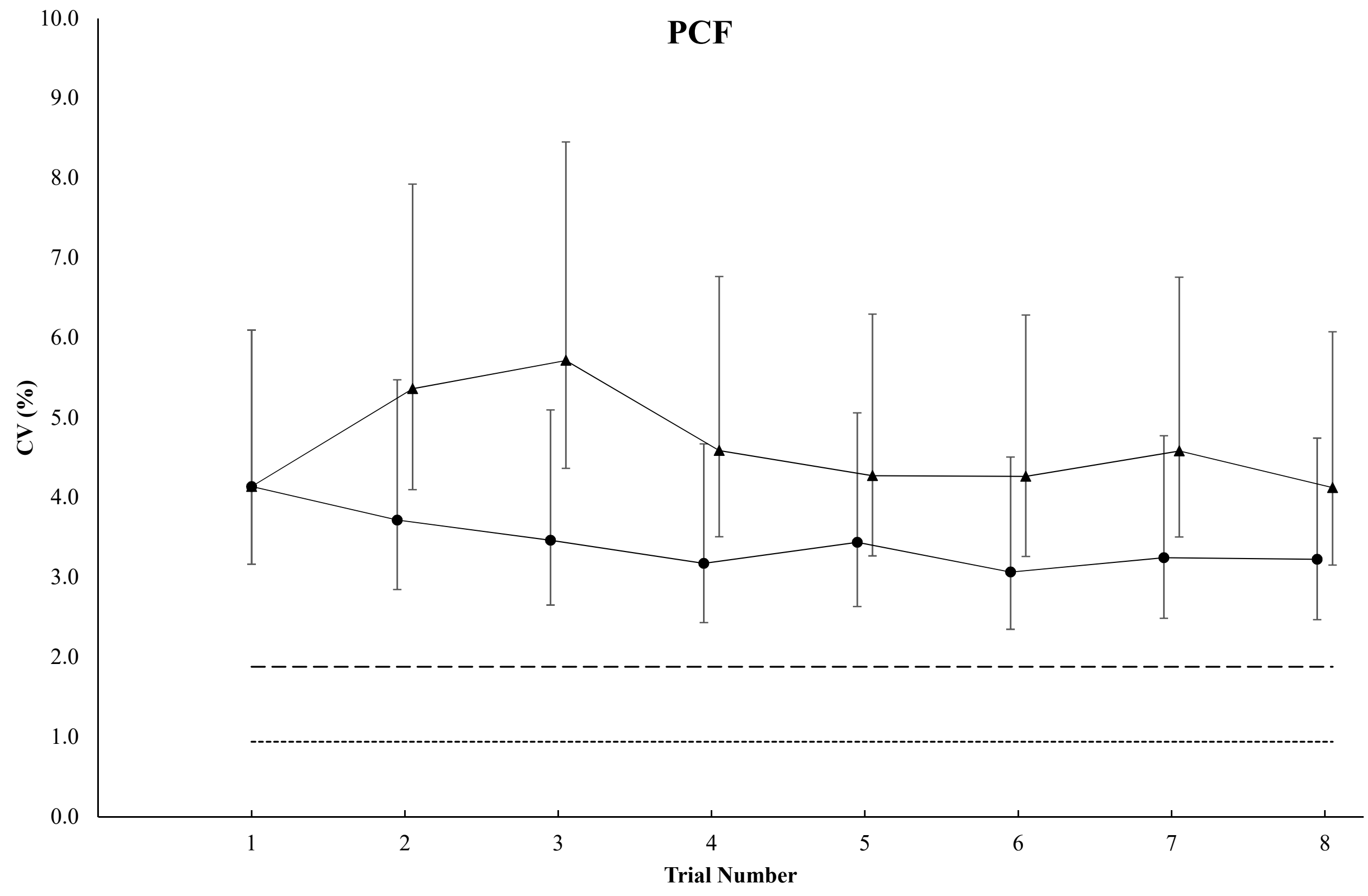




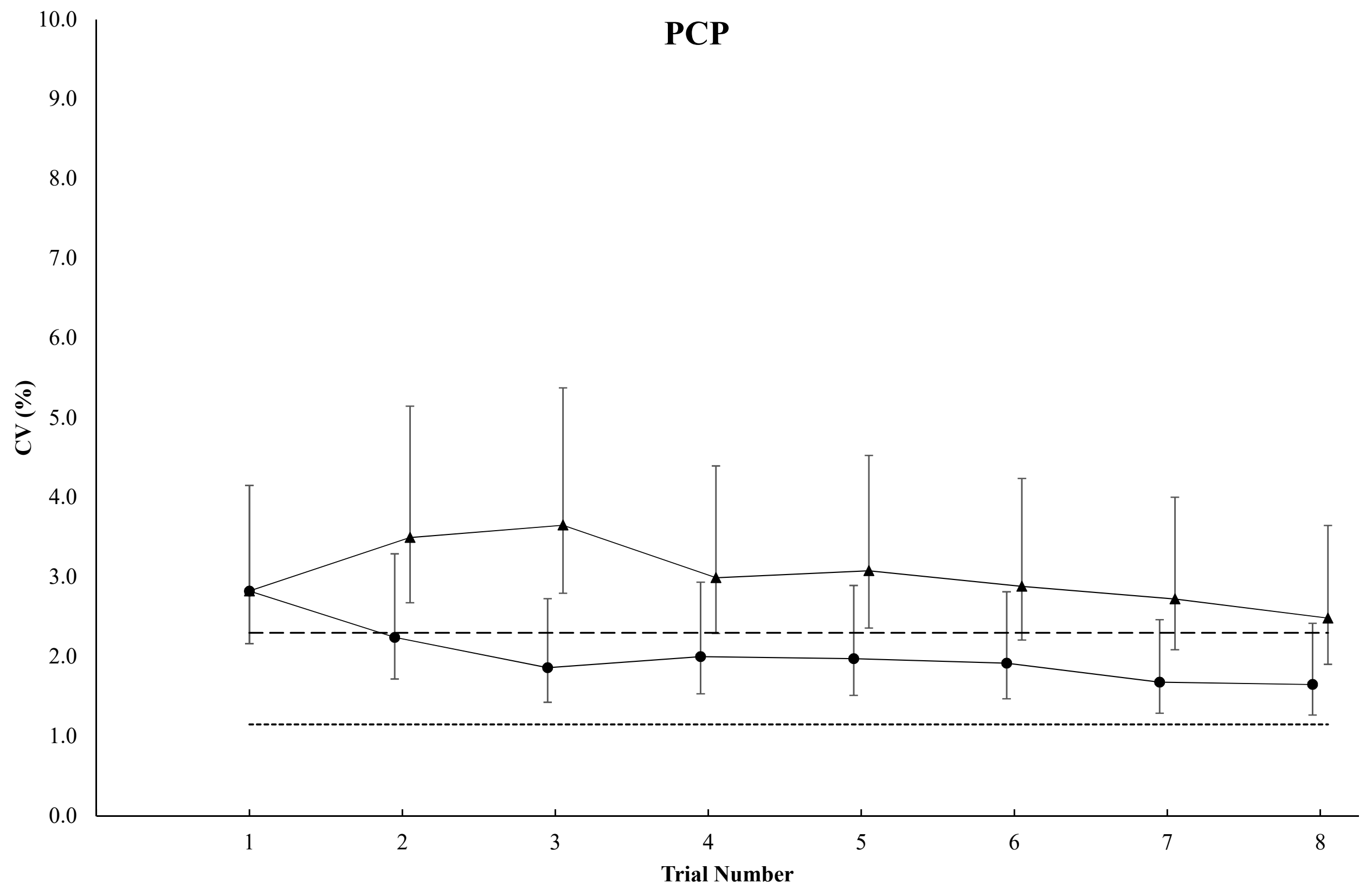




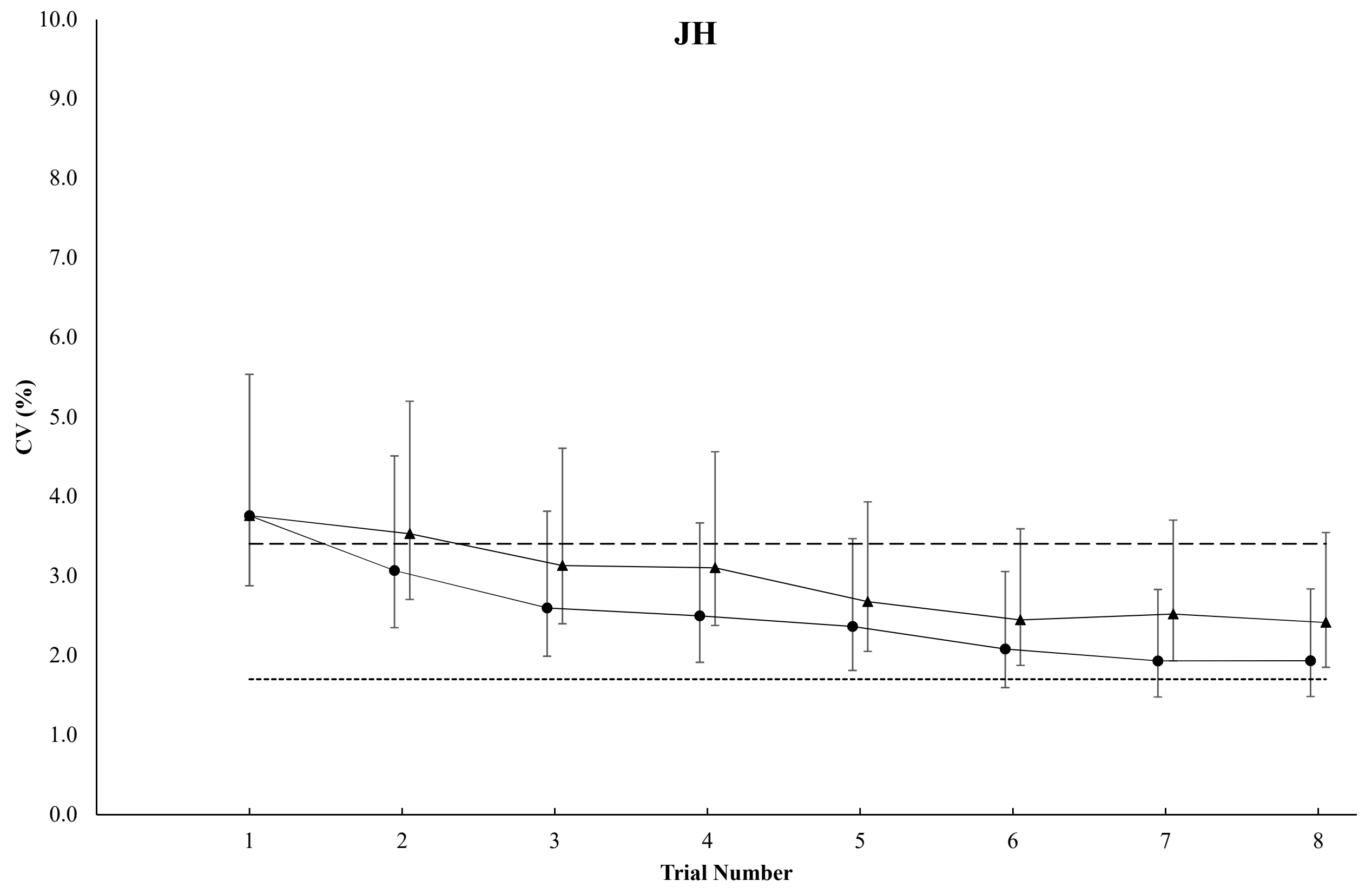




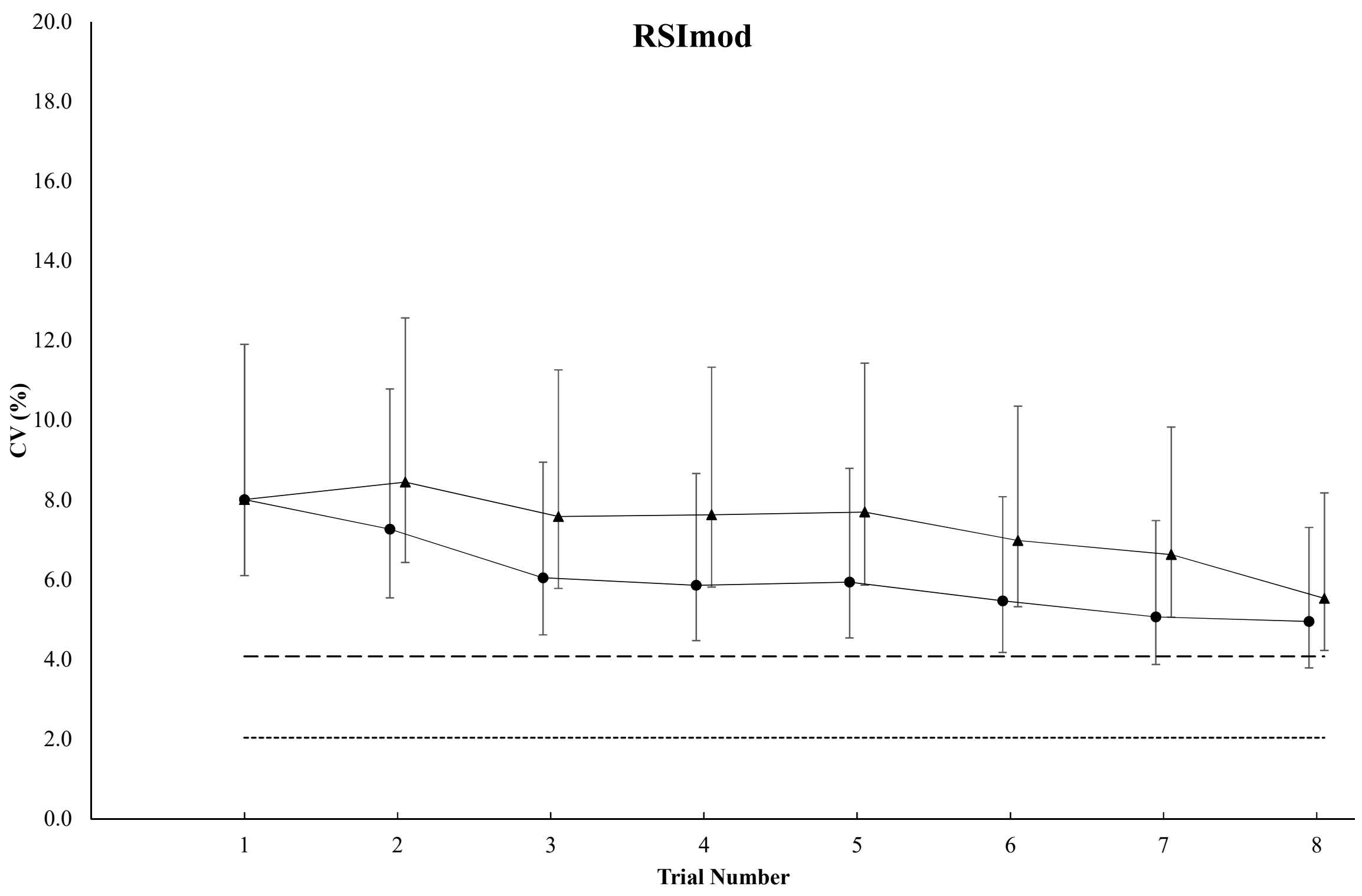




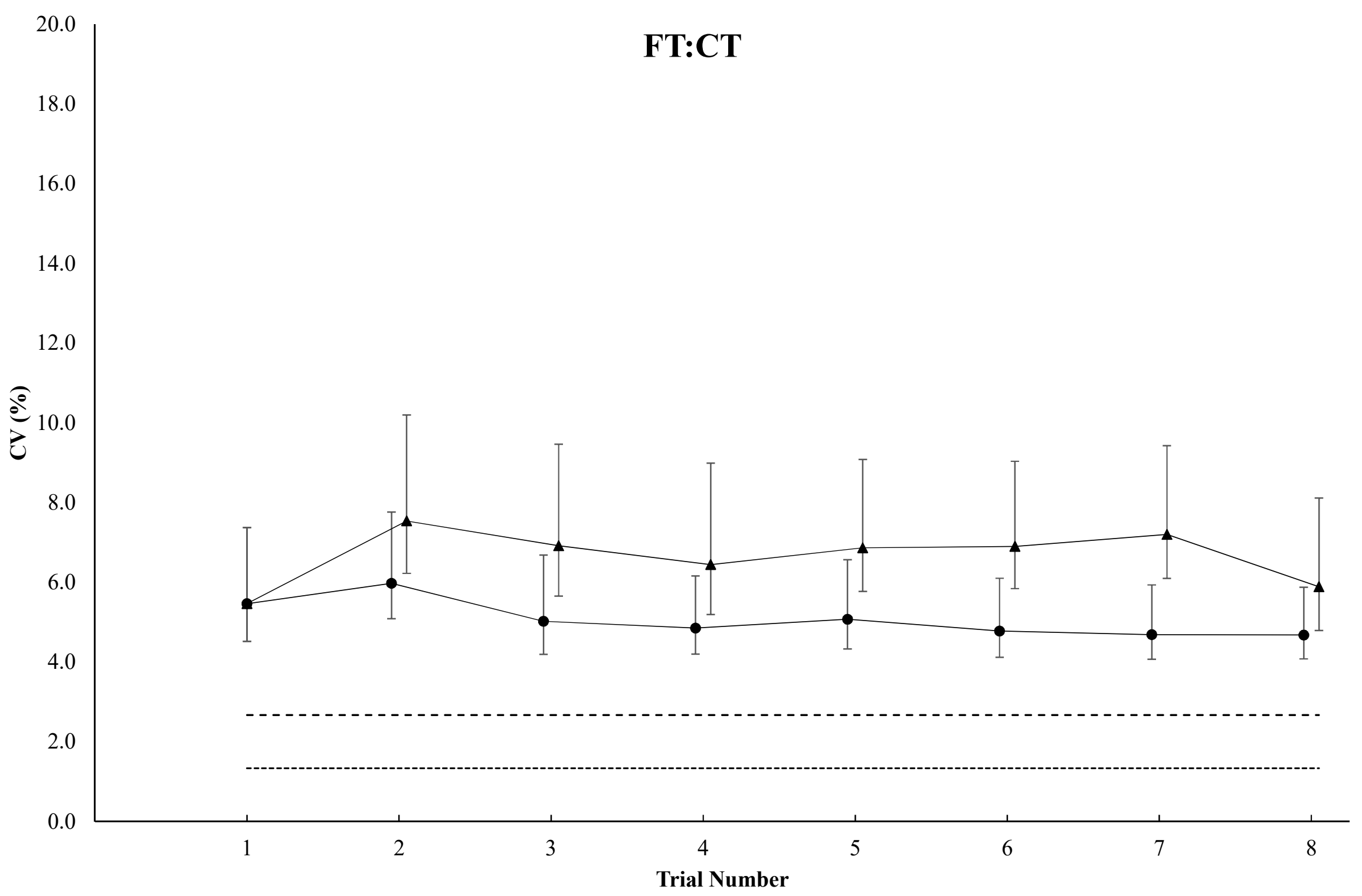




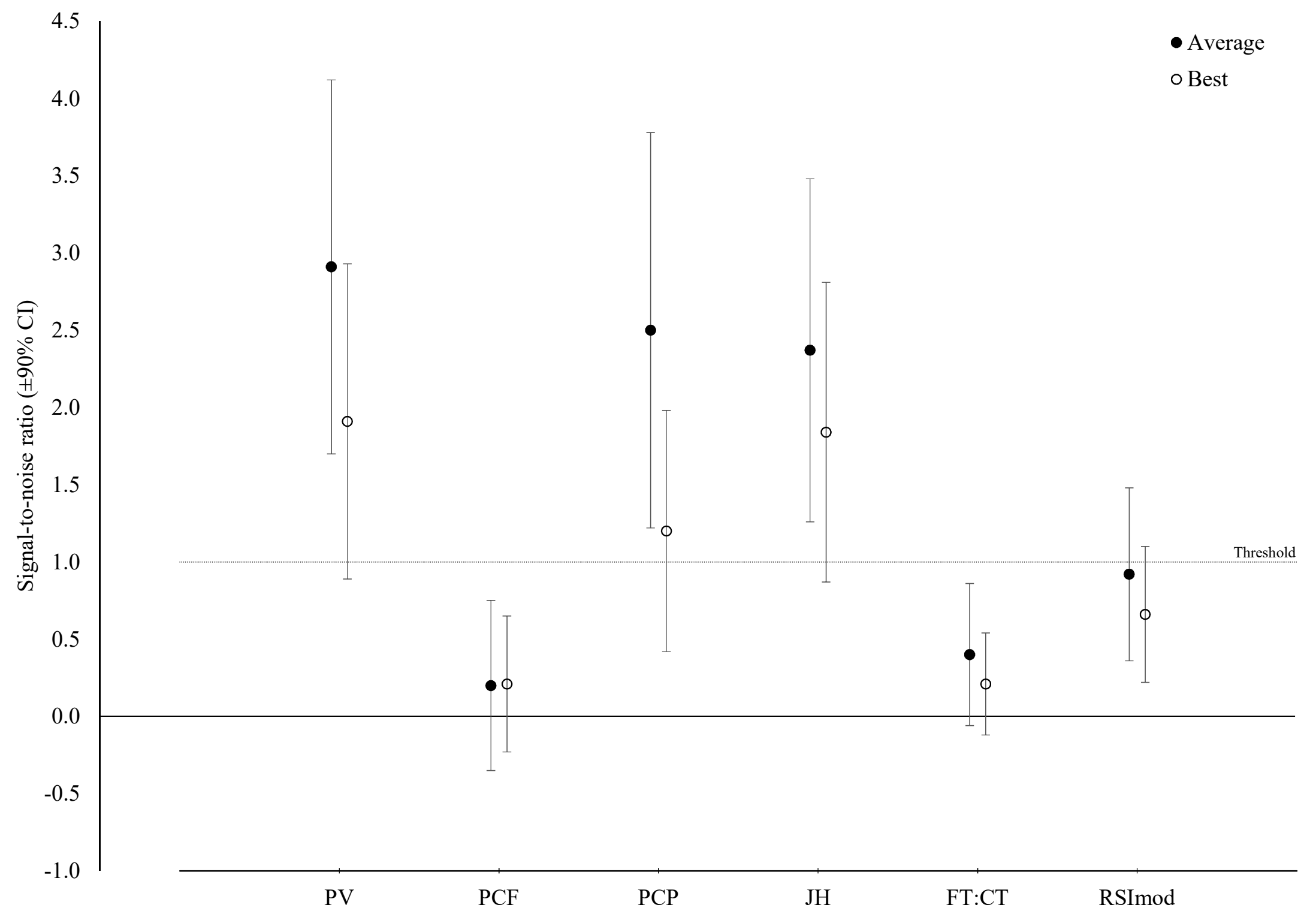

